\title{
Towed-array beamforming during ship's manoeuvring
}

\author{
P.Felisberto \\ S.M. Jesus
}

\begin{abstract}
Towed hydrophone arrays are commonly used for determining the spatial characteristics of the underwater acoustic field. The assumption that the hydrophones lie in a straight and horizontal line is often made when beamforming the hydrophone outputs. However, due to tow vessel motion, ocean swells and currents the array adopts a nonlinear shape and the beamformer output is degraded. To estimate the positions of the hydrophones an array was instrumented with a set of positioning sensors: compasses, tiltmeters, accelerometers and pressure gauges. The authors present the array deformations recorded at sea when the tow vessel is turning and along straight-line tracks. The influence of the observed deformations on the performance of the conventional beamformer output is discussed and illustrated with simulated and real acoustic data.
\end{abstract}

\section{Introduction}

Towed arrays are widely used in underwater acoustics for estimating the horizontal directionality of the acoustic field. Fast plane-wave beamforming algorithms require the assumption that the array is straight and horizontal. The underlying justification is that the use of an equispaced and straight-line array allows to important algorithmic simplifications leading to a dramatic increase of computing efficiency. Unfortunately, this assumption is not always fully verified in practice. In fact due to tow ship speed and course fluctuations, underwater currents, swell and other effects the array shape is often far from linear. It is true that the deviations from the linear shape are also dependent on the array construction parameters like length, thickness, number of mechanical sections and him liaisons. When the array deviates from the assumed linear shape, the beamformer output performance is degraded with bearing estimation errors, loss of beam power and increased sidelobe levels. However, this problem can be avoided if the hydrophone positions can be estimated and the respective corrections introduced in the beamformer.

() IEE, 1996

IEE Proceedings online no. 19960492

Paper first received 29th September 1995 and in revised form 21st March 1996

The authors are with UCEH - University of Algarve, Campus de Gambelas, PT-8000 Faro, Portugal
Generally, two types of method were used to estimate the acoustic sensor positions on a perturbed array. In the first type, the array is instrumented with positioning sensors such as compasses, tiltmeters, pressure gauges and then some interpolation scheme is used to determine the hydrophone positions [1]. Methods of the second kind are known as data driven since they estimate the hydrophone positions from the acoustic data itself. These methods do not need any additional positioning sensors mounted on the array, but array shape estimation is, in these cases, highly time consuming and sometimes sources at known far-field locations are needed $[2-7]$.

In this paper we estimate hydrophone array deformations based on the information recorded at sea with a $156 \mathrm{~m}$-length array instrumented with several positioning modules. The nonacoustic data and the interpolation scheme used to estimate the hydrophone positions is presented. Beamformer outputs are compared in different tow ship's manoeuvring situations to analyse the influence of the array deviations from linearity. This work also addresses the problem of estimating the acoustic field directionality in the full $0-360^{\circ}$ range.

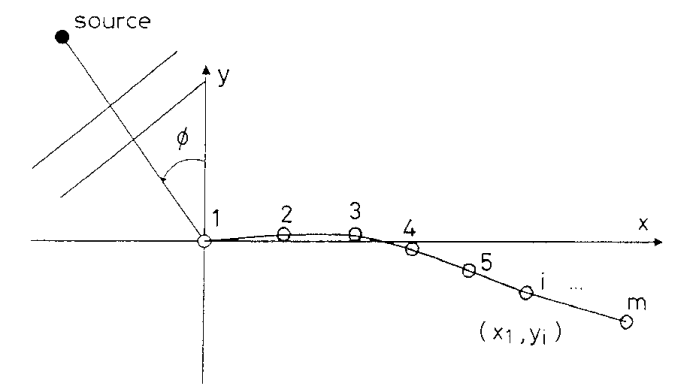

Fig. 1 Amay axis system orientation

\section{Beamforming of deformed towed array}

Beamforming is a well known technique to observe the directionality of the acoustic field sampled by an hydrophone array. To observe signals from a given direction the beamformer coherently adds the hydrophone outputs by adjusting their relative delays for that direction. Frequently, beamforming is performed in the frequency domain, in which case the relative delays are replaced by the equivalent phases. Consider a plane wave impinging at bearing $\phi$ on a towed array with $M$ hydrophones uniformly spaced at $d$ metres as shown in Fig. 1. Our interest concentrates on the azimuthal bearing, thus only the deformations in the $x y$-plane are considered. In the frequency domain, the 
beamforming operation for a given $l$ frequency bin can be written as [3]

$$
G_{l, \phi}=\sum_{m=0}^{M-1} X_{l, m} \exp -j \frac{\omega_{l}}{c}\left\{x_{m} \sin \phi-y_{m} \cos \phi\right\}
$$

where $X_{l, m}$ denotes the $\omega_{l}$ frequency component of the observation at hydrophone $l, c$ is the sound speed, $M$ is the number of sensors and $\left(x_{m}, y_{m}\right)$ is $m$ th hydrophone position. Source bearing is estimated by finding the value of $\phi$ that maximises eqn. 1. Generally, an implementation of eqn. 1 is computationally intensive but it can be enhanced if the assumption that $x_{m}=m d$ is made (i.e. assuming that the disturbance along the $x$ axis can be neglected). Defining the normalised spatial frequency (or wavenumber) $k$ as

$$
k=-\frac{\omega_{l} d M}{c 2 \pi} \sin \phi \quad-\pi / 2<\phi \leq \pi / 2
$$

Thus, rewriting $G$ as function of wavenumber $k$ and using the approximation $x_{m}=m d$

$$
\begin{aligned}
G_{l, k}^{P}=\sum_{m=0}^{M-1} & X_{l, m} \exp -j\left\{\frac{k m 2 \pi}{M}\right\} \\
& \quad \times \exp -j\left\{y_{m} \sqrt{\left(\frac{\omega_{l}}{c}\right)^{2}-\left(\frac{k 2 \pi}{d M}\right)^{2}}\right\} y \geq 0
\end{aligned}
$$

$$
\begin{aligned}
G_{l, k}^{N}=\sum_{m=0}^{M-1} X_{l, m} \exp -j\left\{\frac{k m 2 \pi}{M}\right\} & \\
& \quad \times \exp +j\left\{y_{m} \sqrt{\left(\frac{\omega_{l}}{c}\right)^{2}-\left(\frac{k 2 \pi}{d M}\right)^{2}}\right\} y \leq 0
\end{aligned}
$$

These expressions can be implemented more efficiently than eqn. 1 if some symmetries and similarities are explored. From this point of view the most favourable case occurs when the array is straight and horizontal (all $y_{m}=0$ ) in which case an FFT algorithm can be applied. To have an idea of the quality of the approximation, a comparative study between the outputs from the approximated method (eqns. 3 and 4 ) and the generalised method (eqn. 1) has been made. This study showed that the approximated beampattern could not be distinguished from the true beampattern for sources at or near broadside and showed a maximum error of $5 \mathrm{~dB}$ for sources at or near endfire. That behaviour is approximately constant for any frequency below the maximum array working frequency.

A well known drawback of the straight-line array assumption is the left-right ambiguity. This ambiguity arises because the relative delays between sensors for a chosen source bearing are the same regardless of the source's left-right position. When the array is deformed this no longer happens and therefore the ambiguity problem can be solved. Analysing $G^{P}$ and $G^{N}$, observe that the joining maximum occurs in $G^{P}$ if the source is in the right side of the array. The opposite happens for $G^{N}$ that peaks for a source in the left side of the array.

\section{Estimating array deformation}

A sketch of the array used in the experiment is depicted in Fig. 2. The array has 40 uniformly spaced hydro- phones at $4 \mathrm{~m}$, placed in three acoustic mechanical sections and a tail element. To determine the hydrophone positions the array was instrumented with the following

\begin{tabular}{|c|c|c|c|c|c|}
\hline $32 \mathrm{~m}$ & $32 m$ & $32 m$ & $32 \mathrm{~m}$ & $32 m$ & $32 \mathrm{~m}$ \\
\hline$D P$ C-T-A@ & C-T-Aid & C-T-A & C-T-AOA & C-T-AO & P-C-T- \\
\hline
\end{tabular}
set of positioning sensors:

- six compasses (C1 to $\mathrm{C} 6)$

- six tiltmeters (T1 to T6)

- six accelerometers (A1 to A6)

- two pressure gauges

Fig. 2 Towed array configuration and positioning modules

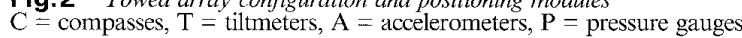

The pressure gauges were installed one in the array head and the other in the array tail. The compass $\mathrm{Cl}$, the tiltmeter $\mathrm{T} 1$ and the accelerometer $\mathrm{Al}$ that form the first positioning module were installed nearby the hydrophone closest to the ship (no. 1). That hydrophone was chosen as the origin of the cartesian positioning system. In this system, it is assumed that the $x$ axis and the first compass direction are colinear. The absolute angles measured by the others compasses ( $\mathrm{C} 2$ to $\mathrm{C} 6$ ) are transformed in relative angles to the $x$-axis by subtracting the values from compass $\mathrm{C} 1$. The positioning modules (each composed of one compass, one tiltmeter and one accelerometer) were placed at $32 \mathrm{~m}$ intervals. To estimate the hydrophone's positions in between two adjacent modules a linear interpolation scheme with a $0.5 \mathrm{~m}$ griding was used. Consider the vector $\left(c_{1}, c_{2}, \ldots, c_{N}\right)$ as the interpolated compass values and the vector $\left(t_{1}, t_{2}, \ldots, t_{N}\right)$ as the interpolated tiltmeter values; $N$ is the number of interpolation samples. To estimate the three dimensional coordinates $x_{i}, y_{i}, z_{i}$; $i=1, \ldots, N$, the following expressions were used

$$
\begin{aligned}
& x_{i}=x_{i-1}+\delta \cos \phi_{i} \cos \lambda_{i} \\
& y_{i}=y_{i-1}+\delta \cos \phi_{i} \sin \lambda_{i} \\
& z_{i}=z_{i-1}+\delta \sin \phi_{i}
\end{aligned}
$$

where $x_{1}=0, y_{1}=0, z_{1}=0, \delta=0.5 \mathrm{~m}$ is the interpolation interval and the angles were computed using

$$
\begin{aligned}
\phi_{i} & =-\frac{\left(t_{i}+t_{i-1}\right)}{2} \\
\lambda_{i} & =-\frac{\left(c_{i}+c_{i-1}\right)}{2}
\end{aligned}
$$

The hydrophone positions are a subset of the computed values for $i=1,9,17,25, \ldots$. This is a very simple procedure that can be easily implemented in a realtime system with inexpensive hardware. Note that the $z$-co-ordinate is estimated here, but for our purposes only the $x$ - and $y$-co-ordinates were used when computing the beamformer output described in Section 2. Also, if the absolute depth is needed then these algorithms can be combined with the measurements obtained from the array head and tail pressure gauges. Initially the installation of accelerometers was decided based on the observation that accelerations might be necessary to correct tiltmeter outputs since tiltmeters act like pendulums and are therefore very sensitive to accelerations. During the cruise a strong bias was observed on the accelerometer data and no correction seemed to be necessary on the tiltmeter outputs. In the sequel we concentrate on the compass-tiltmeter data. 


\section{At-sea data acquisition and processing}

\subsection{Tow-vessel navigation data}

The sea trial took place in the Strait of Sicily in the Pantelleria area. Figs. 3-5 give a general view of the tow vessel navigation data during day 04 of March 1994. The ship made two $180^{\circ}$ port turns. At these moments we expect significant disturbances in the array shape. Another navigation parameter that is important in the array deformation is the ship's speed. As can be seen the ship's speed fluctuations are relatively small during straight-line tracks and showed some variability only during the turns.

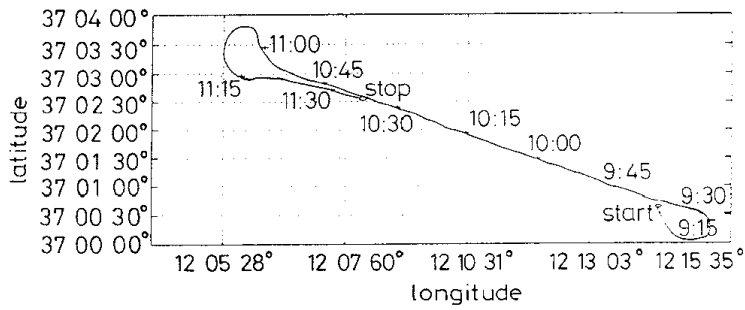

Fig.3 Ship navigation data during sea trial day 04 March 1994: ship track in

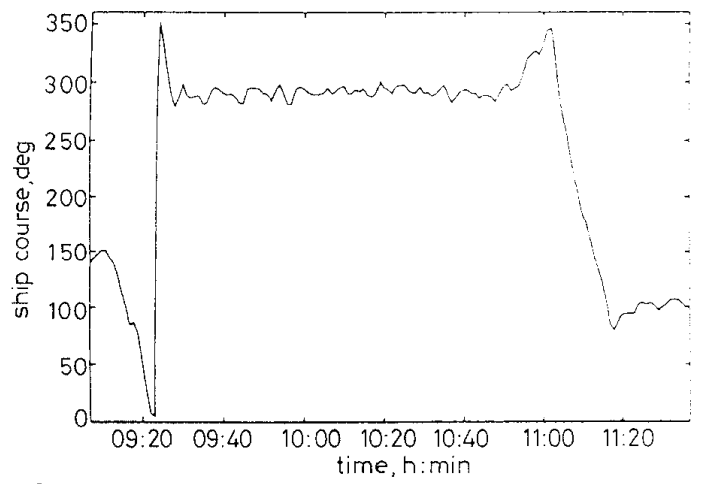

Fig.4 Ship navigation data during sea trial day 04 March 1994: ship course in

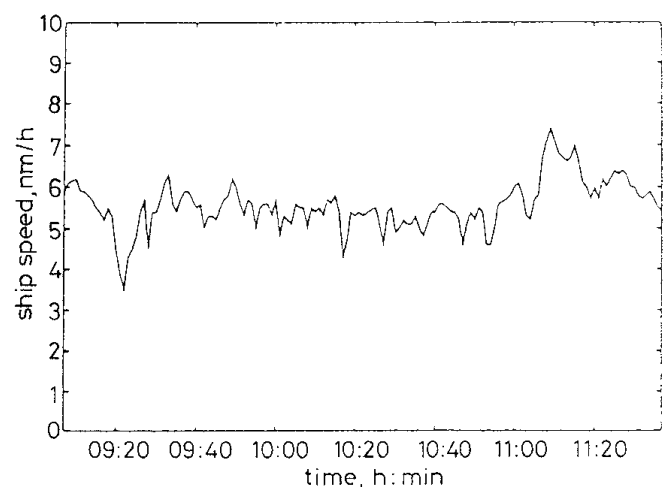

Fig.5 Ship navigation data during sea trial day 04 March 1994: ship ground speed in

\subsection{Array positioning data}

The positioning sensor data was acquired with a sampling rate of one sample per second. Output examples of positioning sensor data for the time interval 09:07 to 11:38 are presented in Figs. $6-8$. Fig. 6 shows the output data for one compass. The compasses diagrams are delayed replicas of the ship's course plot (Fig. 4). Data from one of the tiltmeters are shown in Fig. 7. These data needed to be filtered to eliminate the high frequency components that are possibly due to flow noise. Also, from this plot the moments where the tow vessel turns are perceivable. Fig. 8 presents data from the pressure gauge located in array head, after conversion to depth. From this curve one can easily perceive when ship is turning. The output data from the other pressure gauge (located in the array tail) has a similar evolution with a constant bias (about $10 \mathrm{~m}$ ), even during turns.

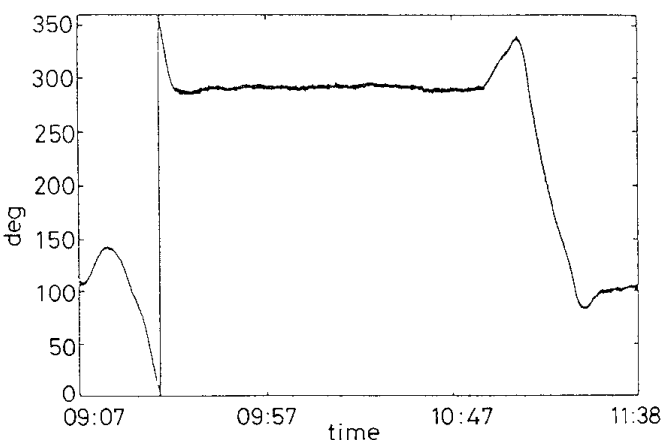

Fig.6 Positioning data: compass

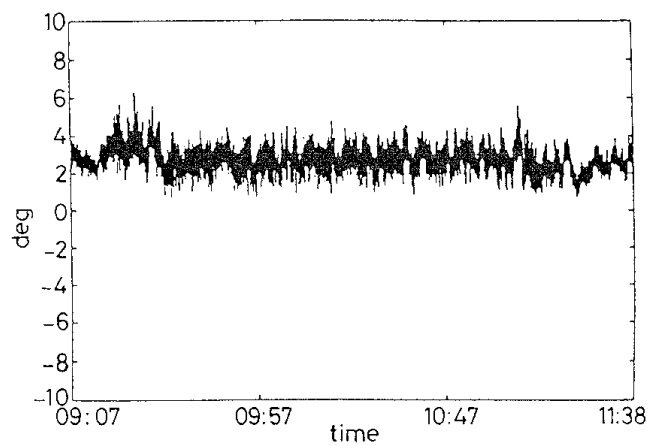

Fig.7 Positioning data: tiltmeter

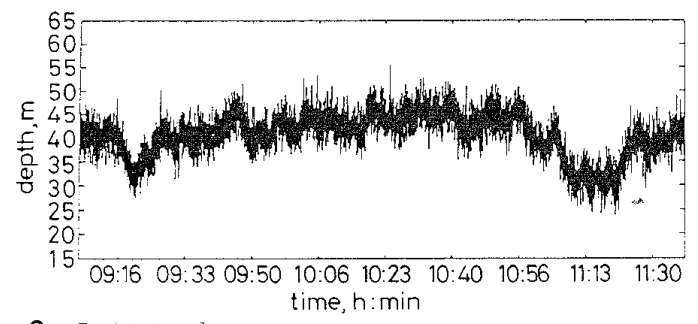

Fig. 8 Positioning data: pressure gauge

\subsection{Estimated array deformation from recorded positioning data}

The array deformation was estimated using the procedure proposed in Section 3. Figs 9 and 10 illustrate a set of representative array shapes recorded before the turn (dotted line), during the turn (dashdot line) and after the turn (dashed line).

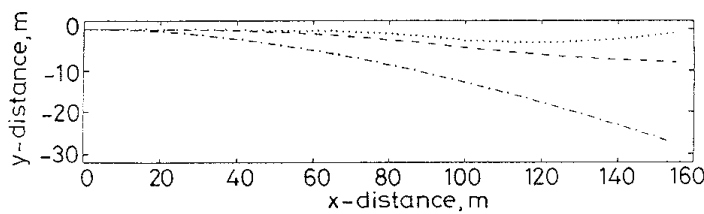

Fig.9 Estimated array deformations in horizontal $X Y$ plane before ship turn; --- during ship turn; - - - after ship turn 


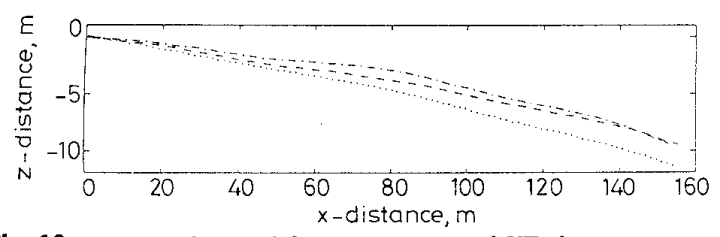

Fig.10 Estimated array deformations in vertical $X Z$ plane before ship turn; -- during ship turn; - - - after ship turn
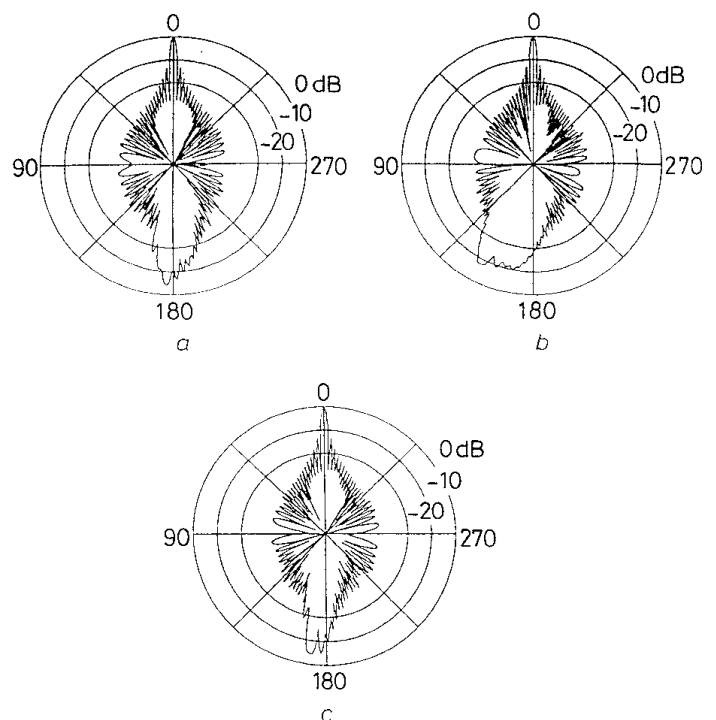

Fig. 11 Beam patterns for a broadside source with real array deformations according to Figs. 9 and 10

(a) Before ship turn (b) During ship turn (c) After ship turn

The spatial filtering characteristics of an array are sometimes illustrated by the beam power pattern. In Fig. 11 the normalised beam power patterns are plotted according to the array deformations of Figs. 9 and 10 for the maximum working frequency of the array $(187.5 \mathrm{~Hz})$ without steering. Depending on the level of array deformation the beam power pattern for the portion of the plane complementary to the source has a lower main lobe but a larger beam width, something like a shade. This is the starting point to solve the right-left ambiguity problem. From these Figures a difference of up to $8 \mathrm{~dB}$ between the main lobe and the shade can be noted. It can be shown that for the array considered and its observed deformations this difference generally increases with array deformation, and the greater values are observed when the source is broadside to the array and its frequency is close to the array maximum working frequency. Since plots in Fig. 11 represent the theoretical beam power patterns for the deformations considered, it is expected that in real world, owing to sensor errors, related array shape estimation errors and other errors, the difference between the main lobe and the shade is somehow smaller than the theoretical.

\subsection{Processing acoustic data}

Real acoustic data has been processed with the beamforming expressions (eqns. 3 and 4), which are approximations of eqn. 1. As an example of the application to field data, a few observations recorded at one minute intervals during the second ship turn between 11:05 and 11:08 a.m. are presented in Figs. 12-23. The sampling frequency was $750 \mathrm{~Hz}$ and the block size used for
FFT computation was equal to 1024 samples. No averaging was done and the number of beams was equal to 128. During that experiment the ship was simultaneously towing an array and an acoustic source emitting tones at 100,120 and $125 \mathrm{~Hz}$. The acoustic field is essentially composed of the towed source signal at bearing $90^{\circ}$ and a strong nearby merchant ship echo, covering the frequency range from 60 to $180 \mathrm{~Hz}$, close to broadside. There are a number of other minor noise sources, possibly owing to distant ships close to endfire.

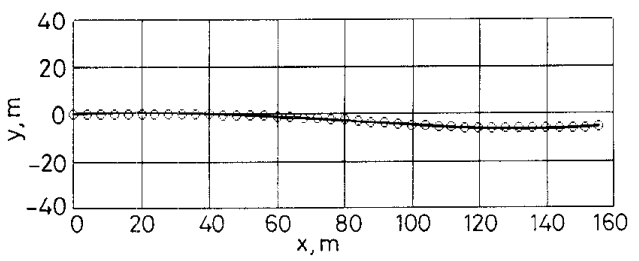

Fig. 12 Field towed-array data at 11:05 am: xy-plane array deformation

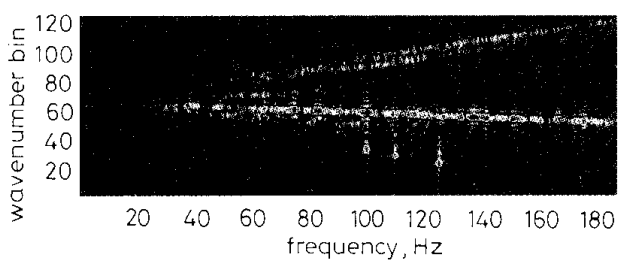

Fig.13 Field towed-array data at 11.05 am: beanformor output for straight-line array
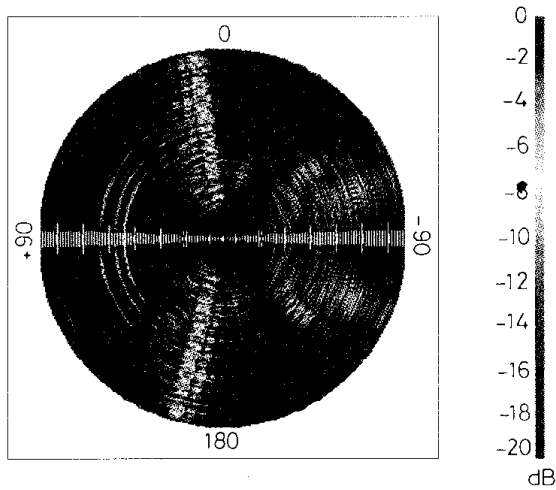

Fig. 14 Beanformer output for $360^{\circ}$ for the arra deformation of Fig. 12, frequency increasing from centre (zero) to border $(187.5 \mathrm{~Hz}$

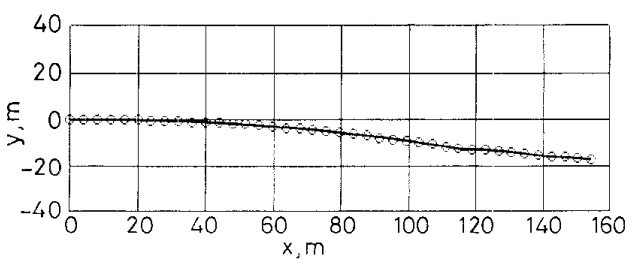

Fig. 15 Field towed-array data at 11.06 am: xy-plane array deformation

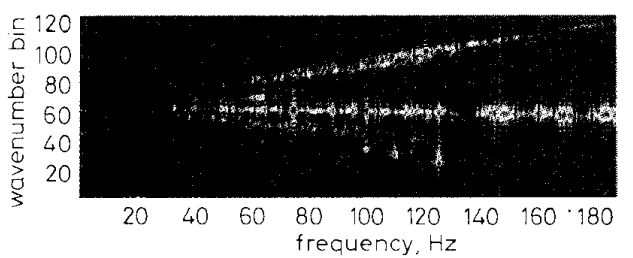

Fig. 16 Field towed-array data at 11:06 am: beamformer output for straight-line array 

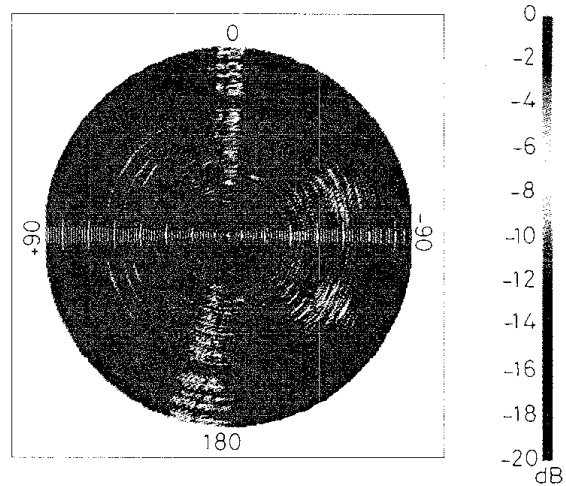

Fig. 17 Beanfomer output for $360^{\circ}$ for the aras defommion of Fig. 15, frequency increasing from centre (zero) to border $(187.5 \mathrm{~Hz}$

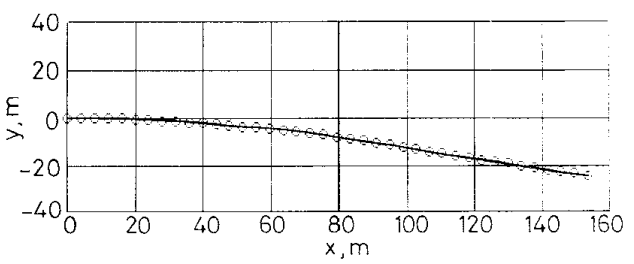

Fig.18 Field towed-array data at 11:07 am: xy-plane array deformation

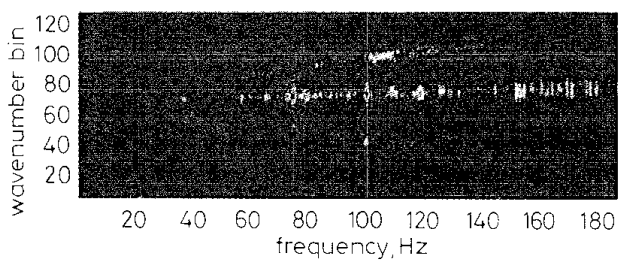

Fig.19 Field towed-array data at 11:07 am: beamformer output for stratght-line array
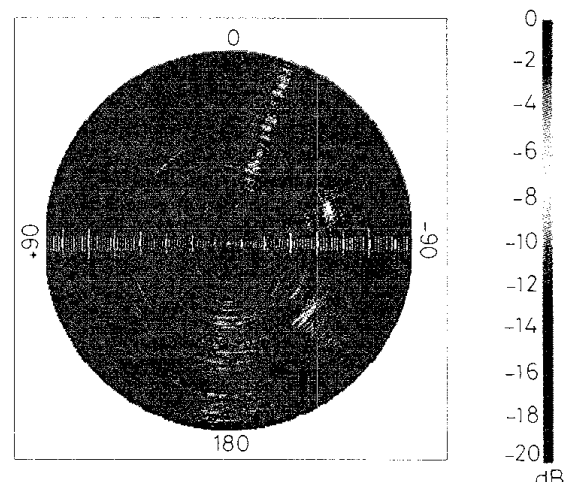

Fig.20 Beamformer output for $360^{\circ}$ for the arroy defomation of Fig. 18, frequency increasing from centre zero to border $187.5 \mathrm{~Hz}$

The sequence of observed array deformations are illustrated in Figs. 12, 15, 18 and 21. One can say that the array deformation is progressively increasing in the $x y$-plane up to $38 \mathrm{~m}$ deviation at the array tail relative to the first hydrophone. Along this sequence, Figs. 13, 16, 19 and 22 show the beamformer output assuming a straight-line array. Figs. 14, 17, 20 and 23 present the beamformer output using eqns. 3 and 4 with the estimated array deformations shown in Figs. 13, 15, 18 and 21 , respectively. In the $360^{\circ}$ plots, frequency increases linearly from zero in the centre to $187.5 \mathrm{~Hz}$ (the maximum working frequency) in the border.
In Figs. 12-14, with an almost straight-line array, the strong ship echo at $10^{\circ}$ can be clearly seen together with the three towed source tones. The $360^{\circ}$ plot Fig. 14 shows that the left-right ambiguity cannot be resolved. Figs. 15-23, with a progressive array deformation, show that the strong ship echo is smeared on Figs. 16, 19 and 22 (that assumes a straight-line array) and the towed source tones cannot be distinguished from background noise. Simultaneously, the $360^{\circ}$ plots show an increasing difference on the acoustic field received from the left and right sides of the array. A close analysis of this difference confirms that the strong ship echo is effectively located starboard at $10,0,-10$ and $-45^{\circ}$ from Figs. 14, 17, 20, 23, respectively.

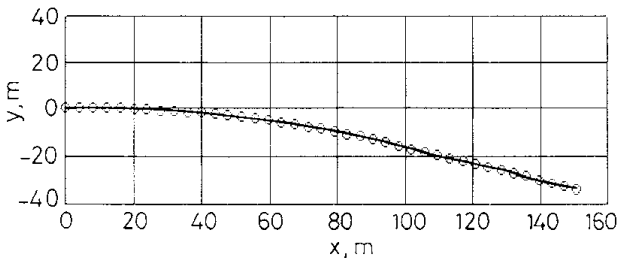

Fig.21 Field torred-array data at 11:08 am: xy-plane array deformation

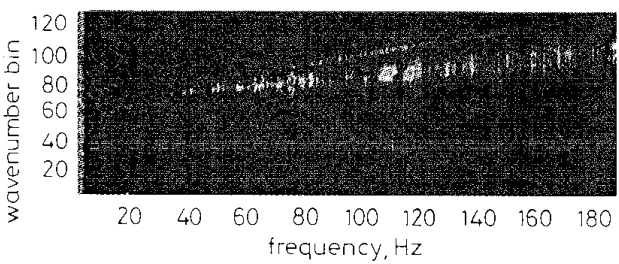

Fig.22 Field towed-array data at 11:08 am: beamfomer output for straight-line array
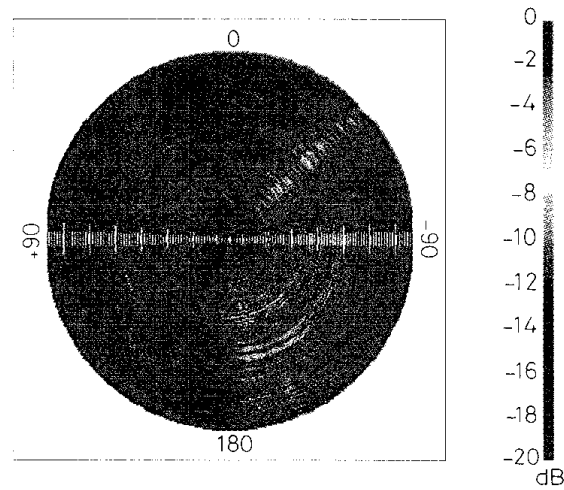

Fig.23 Beamfomer output for $360^{\circ}$ for the array deformation of Fig. 21 frequency increasing from centre (zero) to border $(187.5 \mathrm{~Hz})$

\section{Conclusions}

This paper has shown a number of nonacoustic measurements aimed at estimating towed-array deformations at sea. According to these observations it can be concluded that a towed array is never straight nor horizontal. As expected, this deviation from linearity is higher when the ship turns. In this case the beamformer output is highly reduced, inducing bearing estimation errors, loss of beam power and increased sidelobe levels. This paper presents a simple and fast method to estimate the array deformation based on nonacoustic positioning data recorded in real time and to include the deformation into the beamformer. It is shown that, depending on the deformation of the array, the leftright ambiguity can be resolved. The proposed models 
to estimate the array shape and the acoustic field directionality were successfully applied to real data where right and left sources could be unambiguously located on a $0-360^{\circ}$ ring.

\section{Acknowledgments}

The authors acknowledge A. Kristensen and A. Caiti, scientists in charge, the master and crew of the $\mathrm{R} / \mathrm{V}$ Alliance and the SACLANT Centre Engineering Department for their outstanding respective contributions in the leadership, sea-going operation and equipment preparation before and during the sea trial. The support of E. Dias and E. Coelho from the Hydrographic Institute, Lisbon, on the acquisition of the nonacoustic data is also appreciated. This work was supported by the Marine Science and Technology (MAST II) programme of EC under contract MAS2CT920022.

\section{References}

1 HOWARD, B.E., and SYCK, J.M.: "Calculation of the shape of towed underwater acoustic array', IEEE J. Ocean. Eng., 1992, 17, pp. 193-203

2 BUCKER, H.P.: 'Beamforming a towed line array of unknown shape', J. Acoust. Soc. Amer., 1978, 63, pp. 1451-1454

3 WAHL, D.E.: 'Towed array shape estimation using frequency wavenumber data', IEEE J. Ocean. Eng., 1993, 18, pp. 582-590

4 BOUVET, M.: 'Beamforming of a distorted line array in the presence of uncertainties on the sensor positions', J. Acoust. Soc. Amer., 1987, 81, pp. $1833-1840$

5 FERGUSON, B.G.: 'Sharpness applied to the adaptive beamforming of acoustic data', J. Acoust. Soc. Amer, 1990, 88, pp. 2695-2701

6 QUTNN, B.G., BARRETT, R.F., KOOTSOOKOS, P.J., and SEARLE, S.J.: "The estimation of the shape of an array using a hidden Markov model', IEEE J. Ocean. Eng., 1993, 18, pp. 557564

7 FERGUSON, B.G.: 'Remedying the effects of array shape distortion on the spatial filtering of acoustic data from a line array of hydrophones', LEEE J. Ocean. Eng., 1993, 18, pp. 565-571

8 GAO, S.-W., and GRIFFITHS, J.W.R.: "Experimental performance of high-resolution array processing algorithms in a towed sonar array environment', J. Acoust. Soc. Amer., 1994, 95, pp. $2068-2080$ 